\title{
Hypothesis
}

\section{COVID-19 Tragic Pandemic: Concerns over Unintentional "Directed Accelerated Evolution" of Novel Coronavirus (SARS- CoV-2) and Introducing a Modified Treatment Method for ARDS}

\author{
Ghadimi-Moghadam A. ${ }^{1 \oplus}$, Haghani M.', Bevelacqua J. J.3, \\ Jafarzadeh A. ${ }^{4}$, Kaveh-Ahangar A. ${ }^{5}$, Mortazavi S. M. J. ${ }^{6,7}$, \\ Ghadimi-Moghadam A. ${ }^{8}$, Mortazavi S. A. R. ${ }^{9 * \odot}$
}

\begin{abstract}
Global health authorities are trying to work out the current status of the novel coronavirus (COVID-19) outbreak and explore methods to reduce the rate of its transmission to healthy individuals. In this viewpoint we provide insights concerning how health care professionals can unintentionally shift the novel coronavirus type to more drug-resistant forms. It is worth noting that viruses usually have different sensitivities to physical and chemical damaging agents such antiviral drugs, UV and heat ranging from extremely sensitive (ES) to extremely resistant (ER) based on a bell-shaped curve. Given this consideration, the widespread infection of people with such ER viruses would be a real disaster. Here, we introduce a modified treatment method for COVID-19-associated pneumonia. In this proposed method, COVID-19 patients will receive a single dose of 100,180 or $250 \mathrm{mSv} \mathrm{X}$-ray radiation that is less than the maximum annual radiation dose of the residents of high background radiation areas of Ramsar that is up to $260 \mathrm{mSv}$. In contrast with antiviral drugs, a single dose of either 100,180 or $250 \mathrm{mSv}$ of low LET X-rays cannot exert a significant selective pressure on the novel coronavirus (SARS-CoV-2) and hence does not lead to directed accelerated evolution of these viruses. Moreover, Low Dose Radiation (LDR) has the capacity of modulating excessive inflammatory responses, regulating lymphocyte counts, and controling bacterial co-infections in patients with COVID-19.
\end{abstract}

Citation: Ghadimi-Moghadam A, Haghani M, Bevelacqua JJ, Jafarzadeh A, Kaveh-Ahangar A, Mortazavi SMJ, Ghadimi-Moghadam A, Mortazavi SAR. COVID-19Trag P, Hagin: Con, B and Intucing a Modified Treatment Method for ARDS. J Biomed Phys Eng. 2020;10(2):241-246. doi: 10.31661/jbpe.v0i0.2003-1085.

\section{Keywords}

Coronavirus; X-Rays; Respiratory Distress Syndrome; Radiation Dosage; Selective Pressure; Directed Evolution

\section{Introduction}

$\mathrm{T}$ he new coronavirus has caused thousands of human infections since December 2019, first in China and later in other countries. han, China in late December 2019. After a few days, a novel coronavirus was identified as the causative agent of this mysterious pneumonia [1]. Although the virus has lower fatality, it spreads faster than its ancestors. It is widely believed that the virus originated in bats then transmitted to humans through yet unknown intermediary animals [2]. The
${ }^{1} \mathrm{MD}$, Pediatric Infectious Ward, Yasuj University of Medical Sciences, Yasuj, Iran

${ }^{2} \mathrm{PhD}$, Department of

Radiology, School of Paramedical Sciences, Shiraz

University of Medical Sci-

ences, Shiraz, Iran

${ }^{3} \mathrm{PhD}$, Bevelacqua Resources, Richland, Washington

99352, United States

${ }^{4} \mathrm{PhD}$, Department of Immunology, Faculty of Medicine, Rafsanjan University of Medical Sciences, Rafsanjan, Iran

${ }^{5} \mathrm{MSc}$, Vice-chancellery for

Research, Shiraz Univer-

sity of Medical Sciences,

Shiraz, Iran

${ }^{6} \mathrm{PhD}$, Medical Physics and

Engineering Department,

School of Medicine, Shiraz

University of Medical Sci-

ences, Shiraz, Iran

${ }^{7} \mathrm{PhD}$, Diagnostic Imaging

Department, Fox Chase

Cancer Center, Philadel-

phia, Pennsylvania 19111

United States

${ }^{8} \mathrm{BSc}$, Faculty of Medicine,

Szeged University, Szeged,

Hungary

${ }^{9} \mathrm{MD}$, School of Medicine,

Shiraz University of Medical

Sciences, Shiraz, Iran

*Corresponding author: S. A. R. Mortazavi

School of Medicine, Shiraz University of Medical Sciences, Shiraz, Iran

E-mail: alireza.mortazavi.med@gmail.com

Received: 17 March 2020 Accepted: 23 march 2020 
World Health Organization (WHO) named this infected disease as coronavirus disease 2019 (COVID-19). COVID-19 disease has raised widespread public health concerns around the world [3-5]. COVID-19 is caused by a betacoronavirus named SARS-CoV-2. By affecting the lower respiratory tract, this virus manifests as pneumonia in humans [6]. Studying the initial transmission dynamics of the infection and assessment of the efficiency of control measures can be a crucial step in the evaluation of the likelihood of a sustained transmission to new areas [7]. On March 11, 2020, speaking at the COVID-19 media brief- ing, the WHO Director-General stated "we are deeply concerned both by the alarming levels of spread and severity and by the alarming levels of inaction. We have therefore made the assessment that COVID-19 can be characterized as a pandemic" [8]. Symptoms of the disease sometimes referred to as novel coronavirus pneumonia (NCP), are nonspecific ranging from completely asymptomatic to severe pneumonia and death. Among COVID-19 patients, approximately $80 \%$ of laboratory-confirmed patients showed mild to moderate disease.

Figure 1 shows the concept of selective pressure and directed evolution. Regardless of the

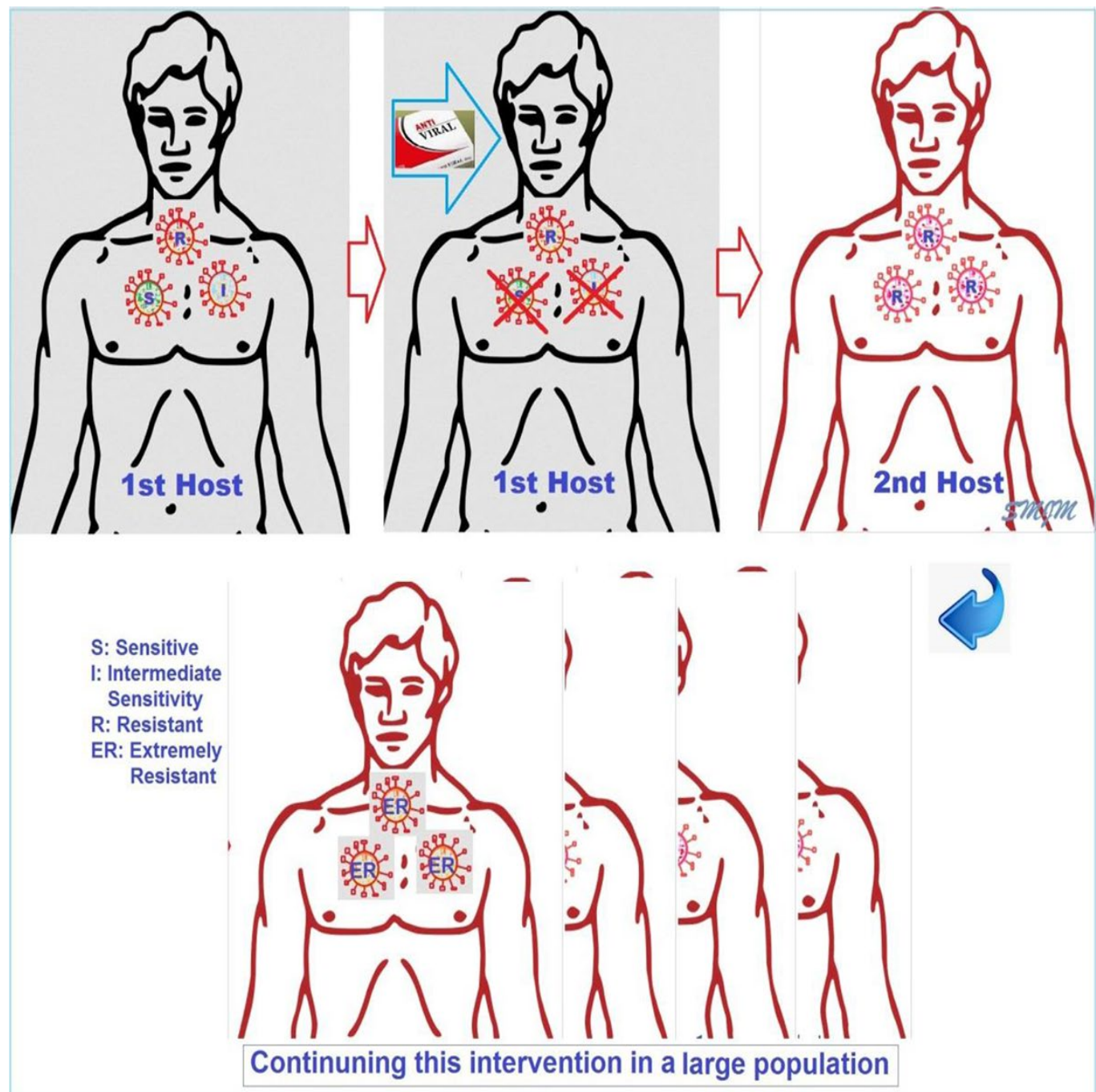

Figure 1: How the two phenomena of selective pressure and directed accelerated evolution can shift the population of viruses to extremely resistant ones. 
Concerns Over Selective Pressure on SARS-CoV-2

validity of this idea that there are two different types of the new coronavirus that cause infections worldwide, even if we assume that there is only one type, within this type different viruses would show differential levels of sensitivity to antiviral drugs, UV, heat, etc. Figure 2 shows the initial heterogeneity of the viruses in terms of their sensitivity to agents such as antiviral drugs. As shown in the Figure 2, using antiviral drugs, the two phenomena of selective pressure and directed evolution can shift the population of viruses to extremely resistant ones. Given this consideration, based on a bell-shaped pattern, different COVID-19 viruses show levels of sensitivity from extremely sensitive to extremely resistant. However, when we expose these viruses to a deleterious agent (antiviral drugs, UV, heat, etc.), the sensitive (S), and moderately sensitive (I) viruses become inactive (elimination by directed evolution) and active resistant (R) viruses can proliferate and transmit the disease to others. By continuing this process for a large group of people, resistant virus gradually becomes Very Resistant (VR) or even Extremely Resistant (ER). Given this consideration, health care providers can unintentionally shift the novel coronavirus forms to more drug-resistant virus types. It is worth noting that new studies show that commonly used antiviral drugs such as neuraminidase inhibitors (oseltamivir, paramivir, zanamivir, etc.), ganciclovir, acyclovir and ribavirin are not valid for 2019-nCoV [9]. Some evidence shows that remdesivir, lopinavir/ritonavir, lopinavir/ritonavir combined with interferon- $\beta$, convalescent plasma, and monoclonal antibodies are possibly effective for 2019-nCoV. However, their efficacy and safety for treatment of 2019-nCoV pneumonia patients should be further evaluated by future clinical trials [9]. Given these considerations, the widespread infection of people with such ER viruses would be a disaster.

In the current situation, considering the possibility of using radiation within the natural background range for treatment coronavirusassociated pneumonitis and prevention of pandemic corona is particularly important. Professor Calabrese, who has served as the editor-in-chief of the renowned "Dose Response" Journal for many years, has published a report titled "How Radiotherapy Was Historically Used to Treat Pneumonia: Could It Be Useful Today?" in the Yale Journal of Biology and Medicine. In his paper, Calabrese reviews a series of studies that showed remarkable results in the treatment of pneumonia by ionizing radiation.

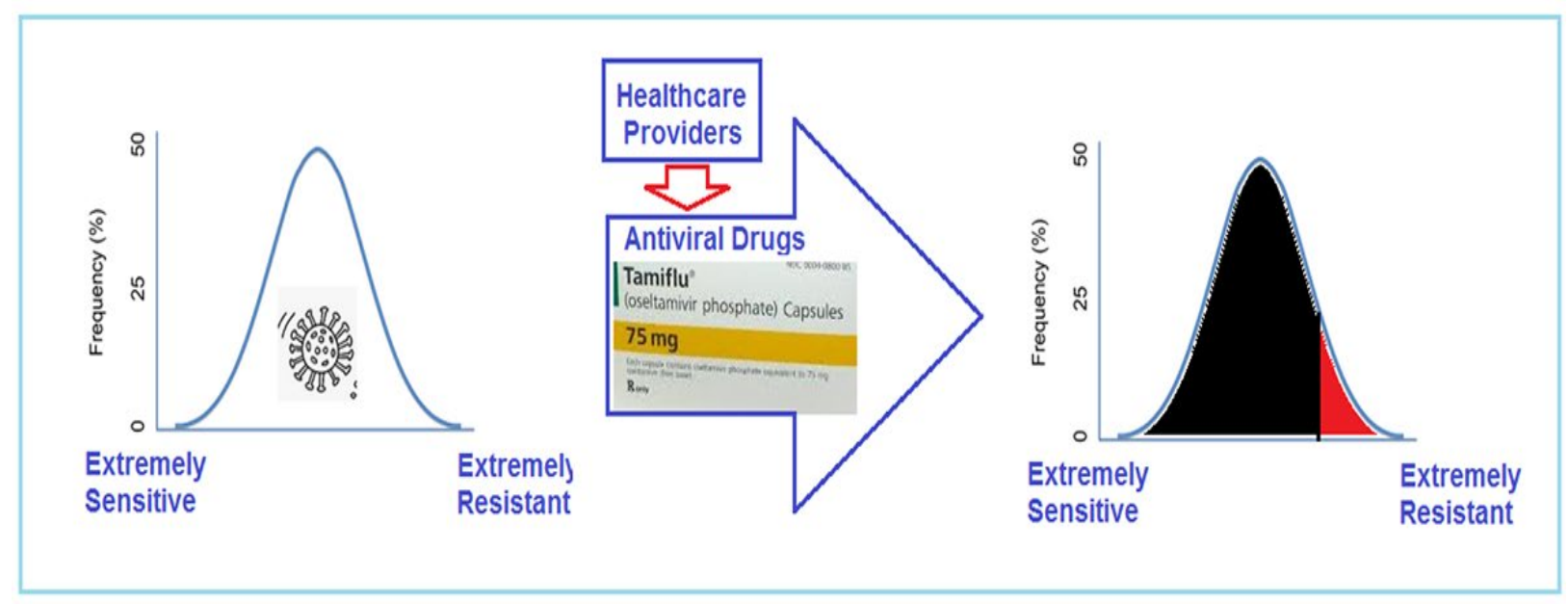

Figure 2: How the two phenomena of selective pressure and directed evolution can shift the population of viruses to extremely resistant ones. 
It should be noted that prior to the pandemic COVID-19, although the radiation dose was relatively low and did not pose a significant risk compared to its therapeutic benefits, this method was not widely used due to radiophobia in the general public and even among scientific community. Mortazavi and his colleagues have been conducting researches on the biological effects of low-doses of ionizing radiation and the health effects of living in areas with high levels of natural radiation since 2002, resulting in the publication of more than 100 articles in internationally accredited journals. Some of these studies have revealed that human exposure to Low Dose Radiation (LDR) can lead to beneficial or stimulatory biological effects, possibly through stimulating the immune system, despite high doses certainly weaken the immune system [10, 11]. Indeed LDR potentiate the fundamental anti-viral immune parameters including natural killer cell activity and interferon production [12]. An accumulating body of evidence supports the concept of LDR-induced immune system enhancement.

In addition to anti-inflammatory effects, accumulating in vitro, animal and human evidence also indicates that LDR may have the capacity to control bacterial pneumonia. Therefore, LDR may also be capable of reducing bacterial co-infections in patients with COVID-19.

It should be noted that immune systemrelated parameters may contribute to the development of the COVID-19-associated complications. Elevated levels of numerous proinflammatory cytokines (such as IFN- $\alpha$, IFN- $\gamma$, IL-1 $\beta$, IL-2, IL-6, IL-7, IL-10, IL-12, IL-18, IL-33, TNF- $\alpha$, TGF- $\beta$ ) and proinflammatory chemokines (such as CCL2, CCL3, CCL5, CXCL8, CXCL9 and CXCL10) were detected in patients with severe COVID-19 $[13,14]$. This phenomenon is called cytokine storm that can promote viral replication and enhance inflammatory-mediated lung damage which lead to other complications such as acute respiratory distress syndrome (ARDS), pneumonitis, shock, respiratory failure, organ failure and possibly death [13]. Cytokine storm, is a deadly uncontrolled systemic inflammatory response resulting from the release of great amounts of pro-inflammatory cytokines, which acts as a major etiologic factor for ARDS development [13, 14]. The lymphopenia and the reduced number of $\mathrm{CD}^{+} \mathrm{T}$ and $\mathrm{CD}^{+} \mathrm{T}$ cells in the peripheral blood of COVID-19 patients were also reported [13, 14]. It has been concluded that similar to SARS and MERS, lymphopenia and cytokine storm may have a major role in the pathogenesis of COVID-19 [13]. The modulatory effects of low-dose ionizing radiation on the production of the proinflammatory cytokines and chemokines were also demonstrated $[15,16]$. Furthermore, it is possible to prevent lymphopenia, prevent the decrease in the number of $\mathrm{CD}^{+} \mathrm{T}$ and $\mathrm{CD} 8^{+} \mathrm{T}$ cells using suitable doses of LDR. Although, radiation using appropriate doses may modulate two major damaging factors (lymphopenia and cytokine storm) in COVID-19, the standardization of the radiation concerning doses, timing and duration need to be clarified in more experiments.

In the current situation as COVID-19 is growing fast in many countries, including Iran, the need for mechanical ventilation systems and ICU facilities, effective management of pneumonia would be crucial in the treatment process of COVID-19 patients. From this perspective, here we introduce a modified effective method for managing pneumonia in COVID-19 patients with the minimal negative effects. In this method, patients will receive a single dose of either 100,180 or $250 \mathrm{mSv}$ $\mathrm{X}$-ray radiation that is less than the maximum annual radiation dose of the residents of high background radiation areas of Ramsar that is up to $260 \mathrm{mSv}$. As shown in Figure 3, to use the advantages of LDR (modulatory effects on the production of the proinflammatory cytokines and chemokines, preventing lymphopenia, preventing the decline of $\mathrm{CD}^{+} \mathrm{T}$ and 


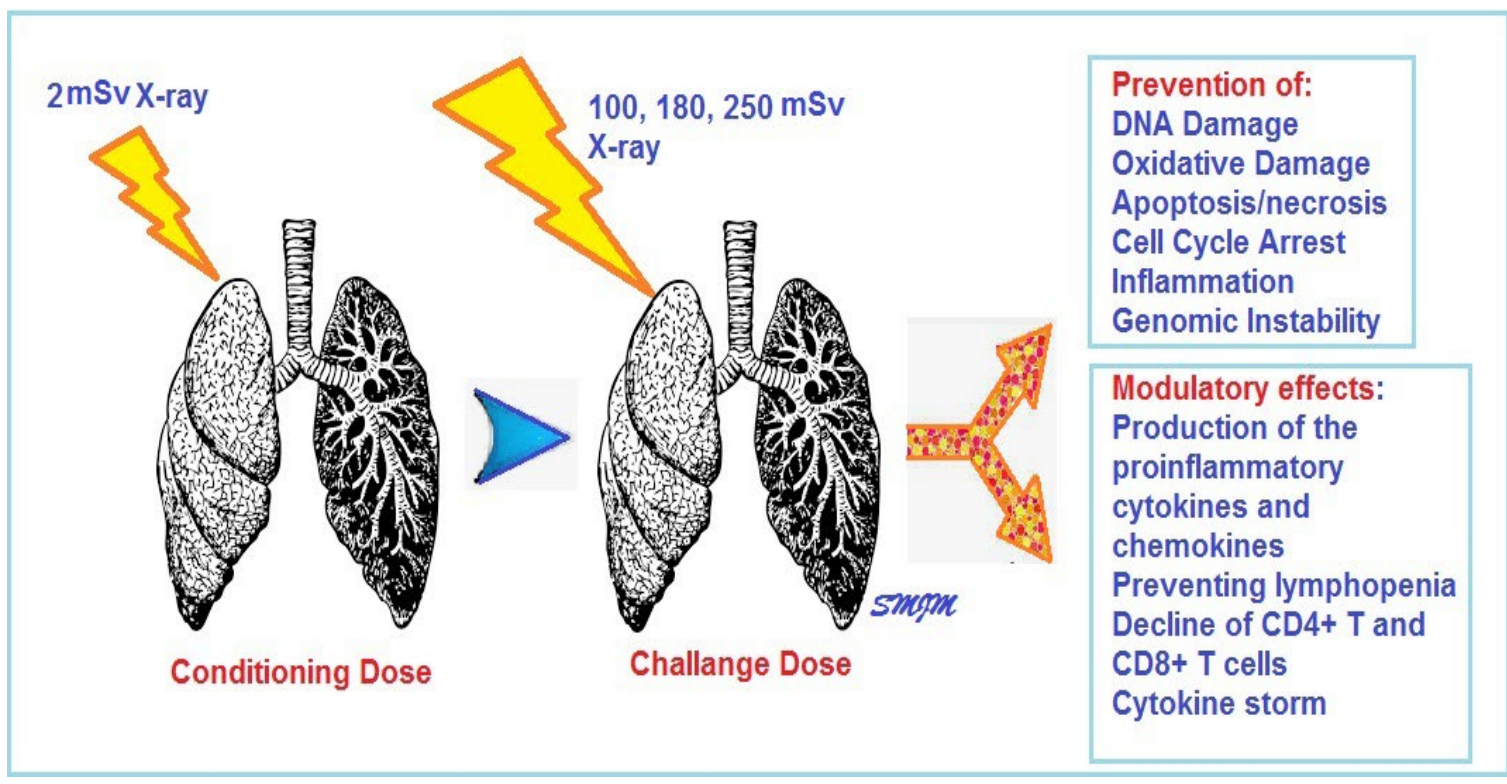

Figure 3: Our proposed treatment plan for COVID-19 associated ARDS.

$\mathrm{CD}^{+} \mathrm{T}$ cells and possible modulation of lymphopenia and cytokine storm in COVID-19 patients), and decreasing the potential risk of the therapeutic dose of X-ray, in our proposed treatment method patients will receive a single adapting dose (a few $\mathrm{mSv}$ of $\mathrm{X}$-ray) before the single dose of 100,180 or $250 \mathrm{mSv}$ X-ray.

\section{Footnotes}

A Ghadimi-Moghadam and M Haghani equally contributed to this work.

\section{Conflict of Interest}

None

\section{References}

1. He F, Deng Y, Li W. Coronavirus Disease 2019 (COVID-19): What we know? J Med Virol. 2020;14(10):25766. doi: 10.1002/jmv.25766. PubMed PMID: 32170865.

2. Singhal T. A Review of Coronavirus Disease-2019 (COVID-19). Indian J Pediatr. 2020;87(4):281-6. doi: 10.1007/s12098-020-03263-6.

3. Sun P, Lu X, Xu C, Sun W, Pan B. Understanding of COVID-19 based on current evidence. J Med Virol. 2020;25(10):25722. doi: 10.1002/jmv.25722.

4. Gao J, Tian Z, Yang X. Breakthrough: Chloroquine phosphate has shown apparent efficacy in treatment of COVID-19 associated pneumonia in clinical studies. Biosci Trends. 2020;14(1):72-3.

5. Tsai J, Wilson M. COVID-19: a potential public health problem for homeless populations. Lancet Public Health. 2020;11(20):30053-0. doi: 10.1016/ S2468-2667(20)30053-0.

6. Sohrabi C, Alsafi Z, O'Neill N, Khan M, Kerwan A, Al-Jabir A, losifidis C, Agha R. World Health Organization declares global emergency: A review of the 2019 novel coronavirus (COVID-19). Int J Surg. 2020;76:71-76. doi: 10.1016/j.jisu.2020.02.034. PubMed PMID: 32112977.

7. Kucharski AJ, Russell TW, Diamond C, Liu Y, Edmunds $J$, Funk $S$, et al. Early dynamics of transmission and control of COVID-19: a mathematical modelling study. Lancet Infect Dis. 2020;11(20):30144-4. doi: 10.1016/S14733099(20)30144-4. PubMed PMID: 32171059.

8. World Health Organization. Rolling updates on coronavirus disease (COVID-19). WHO; 2020.

9. Li H, Wang YM, Xu JY, Cao B. Potential antiviral therapeutics for 2019 Novel Coronavirus. Zhonghua Jie He He Hu Xi Za Zhi. 2020;43(3):170-2.

10. Kumari A, Simon SS, Moody TD, Garnett-Benson C. Immunomodulatory effects of radiation: what is next for cancer therapy? Future Oncol. 2016;12(2):239-56. doi: 10.2217/fon.15.300. PubMed PMID: 26621553. PubMed PMCID: 
PMC5479357.

11. Walle T, Martinez Monge R, Cerwenka A, Ajona D, Melero I, Lecanda F. Radiation effects on antitumor immune responses: current perspectives and challenges. Ther $A d v$ Med Oncol. 2018;10:1758834017742575. doi: 10.1177/1758834017742575. PubMed PMID: 29383033. PubMed PMCID: PMC5784573.

12. Yang $G$, Kong $Q$, Wang $G$, Jin $H$, Zhou $L$, Yu D, et al. Low-dose ionizing radiation induces direct activation of natural killer cells and provides a novel approach for adoptive cellular immunotherapy. Cancer Biother Radiopharm. 2014;29(10):42834. doi: 10.1089/cbr.2014.1702. PubMed PMID: 25402754. PubMed PMCID: PMC4267769.

13. Prompetchara E, Ketloy C, Palaga T. Immune responses in COVID-19 and potential vaccines: Lessons learned from SARS and MERS epidemic.
Asian Pac J Allergy Immunol. 2020. doi: 10.12932/ AP-200220-0772.

14. Li X, Geng M, Peng Y, Meng L, Lu S. Molecular immune pathogenesis and diagnosis of COVID-19. Journal of Pharmaceutical Analysis. 2020. doi: 10.1016/j.jpha.2020.03.001.

15. Schroder S, Kriesen S, Paape D, Hildebrandt G, Manda K. Modulation of Inflammatory Reactions by Low-Dose Ionizing Radiation: Cytokine Release of Murine Endothelial Cells Is Dependent on Culture Conditions. Journal of immunology research. 2018;2018:2856518. doi: 10.1155/2018/2856518.

16. Rodel F, Frey B, Manda K, Hildebrandt G, Hehlgans $S$, Keilholz L, et al. Immunomodulatory properties and molecular effects in inflammatory diseases of low-dose x-irradiation. Front Oncol. 2012;2:120. doi: 10.3389/fonc.2012.00120. PubMed PMID: 23057008. PubMed PMCID: PMC3457026. 